\title{
Safety and Feasibility of Ultrasound-Guided Gastric Access for Percutaneous Transabdominal Gastrostomy Tube Placement
}

\author{
Pratik A. Shukla ${ }^{\text {a }}$, Marcin K. Kolber ${ }^{\mathrm{a}}$, Richard Tapnio ${ }^{\mathrm{a}}$, Adam Zybulewskia, \\ Abhishek Kumar ${ }^{\mathrm{a}}$, Rajesh I. Patel ${ }^{\mathrm{a}, \mathrm{b}}$
}

\begin{abstract}
Background: The aim of the study was to evaluate the safety and feasibility of ultrasound guidance gastric access for percutaneous retrograde transabdominal gastrostomy $(\mathrm{G})$-tube placement.
\end{abstract}

Methods: Twenty-eight patients undergoing 31 percutaneous retrograde transabdominal G-tube placements utilizing ultrasound-guided gastric accesses were retrospectively identified.

Results: All patients had successful placement of G tubes with ultrasound-guided gastric access. There were no cases of aspiration or peritonitis. Average fluoroscopy time was $2.7 \pm 1.4 \mathrm{~min}$ and average radiation dose was $220 \pm 202 \mu \mathrm{Gym}^{2}$.

Conclusions: Ultrasound-guided access for gastrostomy placement is safe and feasible and can be performed with minimal fluoroscopy times resulting in low patient and operator radiation dose.

Keywords: "Push-type" gastrostomy tube; Percutaneous retrograde transabdominal gastrostomy; Ultrasound-guided gastric access; Fluoroscopy time; Radiation dose

\section{Introduction}

Gastrostomy $(\mathrm{G})$ tubes are well established as a means for enteral nutrition in patients with poor or absent oral intake in cases of head and neck malignancy, neurologic deficits (i.e. stroke, neuromuscular disorders, etc.) and diseases of the esophagus

Manuscript submitted January 2, 2019, accepted January 30, 2019

a Division of Vascular and Interventional Radiology, Department of Radiology, Mount Sinai Beth Israel, Mount Sinai Hospital System, Icahn School of Medicine, First Avenue at Sixteenth Street, New York, NY 10010, USA

${ }^{\mathrm{b}}$ Corresponding Author: Rajesh I. Patel, Division of Vascular and Interventional Radiology, Department of Radiology, Mount Sinai Beth Israel, Mount Sinai Hospital System, Icahn School of Medicine, First Avenue at Sixteenth Street, New York, NY 10003, USA. Email: Rajesh.patel@mountsinai.org

doi: https://doi.org/10.14740/gr1136
[1]. G tubes may be placed surgically through a midline laparotomy, but these require general anesthesia and are associated with the highest complication rates [2]. Less invasive methods of percutaneous placement have been developed using endoscopic or radiologic guidance. Percutaneous radiologic placement of a $\mathrm{G}$ tube may be performed in an anterograde transoral ("pull-type") or a retrograde transabdominal ("push-type") fashion [3]. Endoscopic placement of a G tube requires deep sedation and is contraindicated in cases of head and neck malignancy where passage of the endoscope and/or tube into the stomach is not possible, or at risk for G-tube track seeding [4]. Anterograde transoral pull-type image-guided tube placement may not be feasible in these cases for the same reasons.

Placement of a percutaneous radiologic $G$ tube via the retrograde transabdominal route benefits from the use of gastropexy devices in order to juxtapose the stomach to the anterior abdominal wall. This ensures secure access for sequential dilation of a tract to insert the tube through. The placement of gastropexy T-fasteners and a subsequent access needle is typically performed under fluoroscopic guidance after the insufflation of the stomach with carbon dioxide [3]. Although widely accepted, this technique does not account for overlapping structures during gastric puncture and can rarely result in inadvertent through-and-through organ puncture such as colon or liver [1, 5]. In some cases, ultrasound survey prior to gastric puncture has been performed to identify liver margins to avoid a transhepatic access [1].

Gastric access under real-time ultrasound guidance allows for visualization of needle entry into the stomach and can be used as an adjunct to fluoroscopic techniques [6]. Herein, we describe our experience with percutaneous transabdominal Gtube placement using ultrasound guidance for gastric access.

\section{Materials and Methods}

\section{Study population and experimental design}

The protocol for this study was reviewed by the Institutional Review Board. We performed a retrospective review of patients who underwent percutaneous retrograde transabdominal G-tube placements with ultrasound-guided gastric access at our institution from April 2015 to November 2016 using the McKesson Radi- 


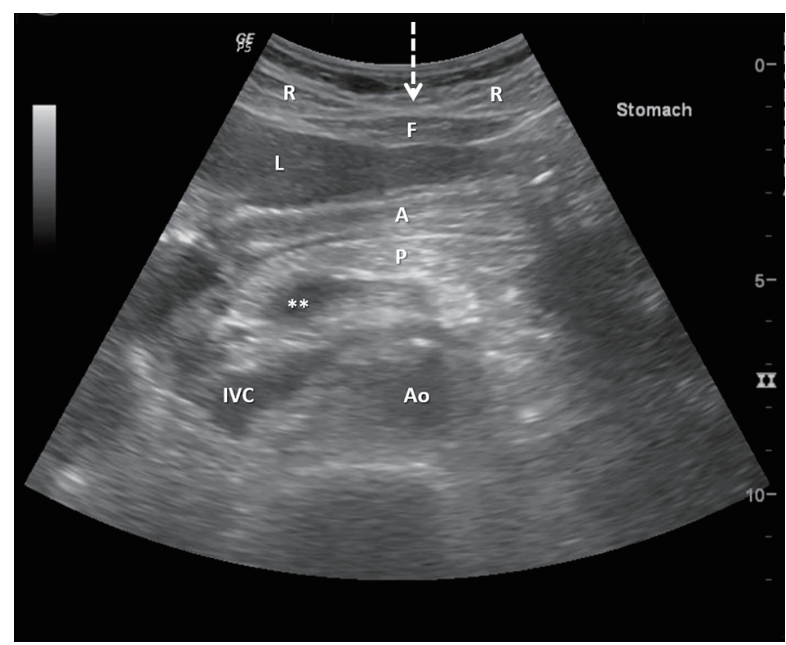

Figure 1. Pre-procedure ultrasound is performed in order to identify the margins of the liver and the location of the stomach and surrounding structures. R: rectus abdominis muscle; $F$ : midline sub-rectus fat pad (arrow demonstrating midline linea alba); L: liver; A: antrum of stomach; P: pancreatic body; IVC: inferior vena cava; Ao: Aorta. ${ }^{* *}$ Portal confluence. The pre-procedure abdominal ultrasound is performed prior to stomach insufflation (which may or may not move the liver margin and allow for access through the linea alba).

ology ${ }^{\mathrm{TM}}$ Picture and Archiving Communication Systems (PACS, McKesson Corporation, San Francisco, CA, USA). Demographic data, pertinent past medical history, procedural information (size of G tube, fluoroscopy times and radiation dose), complications, dislodgements/replacements and follow-up were recorded.

\section{Procedure}

Informed consent was obtained from each patient including discussions pertaining to risks, benefits and alternative procedures. Intravenous antibiotics were given to the patient prior to the procedure (2 g cefazolin). Managed anesthesia care was provided by an attending anesthesiologist when appropriate. Otherwise, moderate sedation was provided with monitored nursing care using intravenous midazolam and fentanyl. Intravenous $1 \mathrm{mg}$ glucagon was administered to decrease bowel motility and permit gastric distension. The patients were placed supine on the fluoroscopy table. In cases where patients did not have an enteric (nasogastric/orogastric) tube, a 4-French (F) angiographic glide catheter was passed through the nares down the esophagus and into the stomach under fluoroscopic guidance to be used for insufflation of the stomach with carbon dioxide. All pertinent available imaging studies (i.e. computed tomography (CT) of the abdomen) were reviewed prior to the procedure to confirm anatomic feasibility of gastrostomy, although having a CT was not a prerequisite. Pre-procedure ultrasound survey of the abdomen was performed using a low frequency curvilinear transducer to again identify liver margins and colon position (Fig. 1).

The stomach was insufflated with carbon dioxide. A percutaneous access window was identified with ultrasound and correlated with fluoroscopy (Fig. 2a). The preferred G-tube access site was midline through the linea alba for patient comfort, if possible. Under ultrasound guidance, the stomach was accessed in two locations lateral to the linea alba on either side to deploy suture anchors (Fig. 2b), also confirmed by fluoroscopy. In the center of the gastropexy site, preferably through the linea alba, an 18-gauge needle was used to access the stomach under ultrasound guidance (Fig. 2c). Access was confirmed with gentle contrast injection through the needle. A 0.035 stiff guide wire was placed into the stomach directed at the fundus (Fig. 2d). The access track was then sequentially dilated and a $\mathrm{G}$ tube was placed into the stomach under fluoroscopic guidance. Most commonly, the tract was dilated up to $20 \mathrm{~F}$ after which a 16-F G tube was placed into the gastric body through a 20-F peel away sheath. The G-tube balloon was inflated to secure the tube in place. Final gentle contrast injection through the tube was performed to confirm placement. The catheter retention disk was advanced to the skin surface. The suture anchors were cut within $24 \mathrm{~h}$ to prevent skin inflammation.

\section{Data analysis/statistical methods}

Technical success was defined as successful placement of a percutaneous $\mathrm{G}$ tube with ultrasound-guided gastric access. Statistical comparison between groups was performed using Student's $t$-test for continuous variables. Two-tailed tests were performed for each scenario and significance level was set at $\mathrm{P}<0.05$. All analyses were performed using Microsoft Office Excel 2007 (Microsoft Corp., Redmond, WA, USA).

\section{Results}

Twenty-eight patients (15 males and 13 females; average age: $60.7 \pm 15.4$ years) underwent 31 ultrasound-guided antral access for transabdominal G-tube placements. Twenty-four patients had head and neck malignancies and required a $\mathrm{G}$ tube for enteral nutrition. Two patients had neurologic deficits and were unable to have oral intake. Two patients required gastric decompression following a Whipple surgery. Average followup time was 125 days.

Technical success was $100 \%$. The most common size $\mathrm{G}$ tube was $16 \mathrm{~F}$ in 21 cases. There were six $18-\mathrm{F}$ and two $20-\mathrm{F}$ and two $12-\mathrm{F}$ gastrostomy tubes placed. There were no procedure-related complications (i.e. bleeding or perforation) according to standard practice guideline [7]. One patient with a 12-F gastrostomy tube placed for gastric decompression after Whipple surgery required upsizing to a $16-\mathrm{F}$ G tube due to repeated clogging. Three patients with $16-\mathrm{F}$ tubes had tubes exchanged due to dislodgement, which was due to inadvertent balloon deflation in all three cases. Three patients had a second $\mathrm{G}$ tube placed after the initial tube was removed for resumption of per oral intake. One patient with an 18-F tube had tube dysfunction due to clogging but was successfully recanalized at bedside with passage of a 0.035 guidewire. One patient had erythema and purulent discharge around the tube site which was managed conservatively with antibiotics. One patient had mild leakage around the tube which resolved with retention disc readjustment. There were no cases of aspiration or peritonitis. 

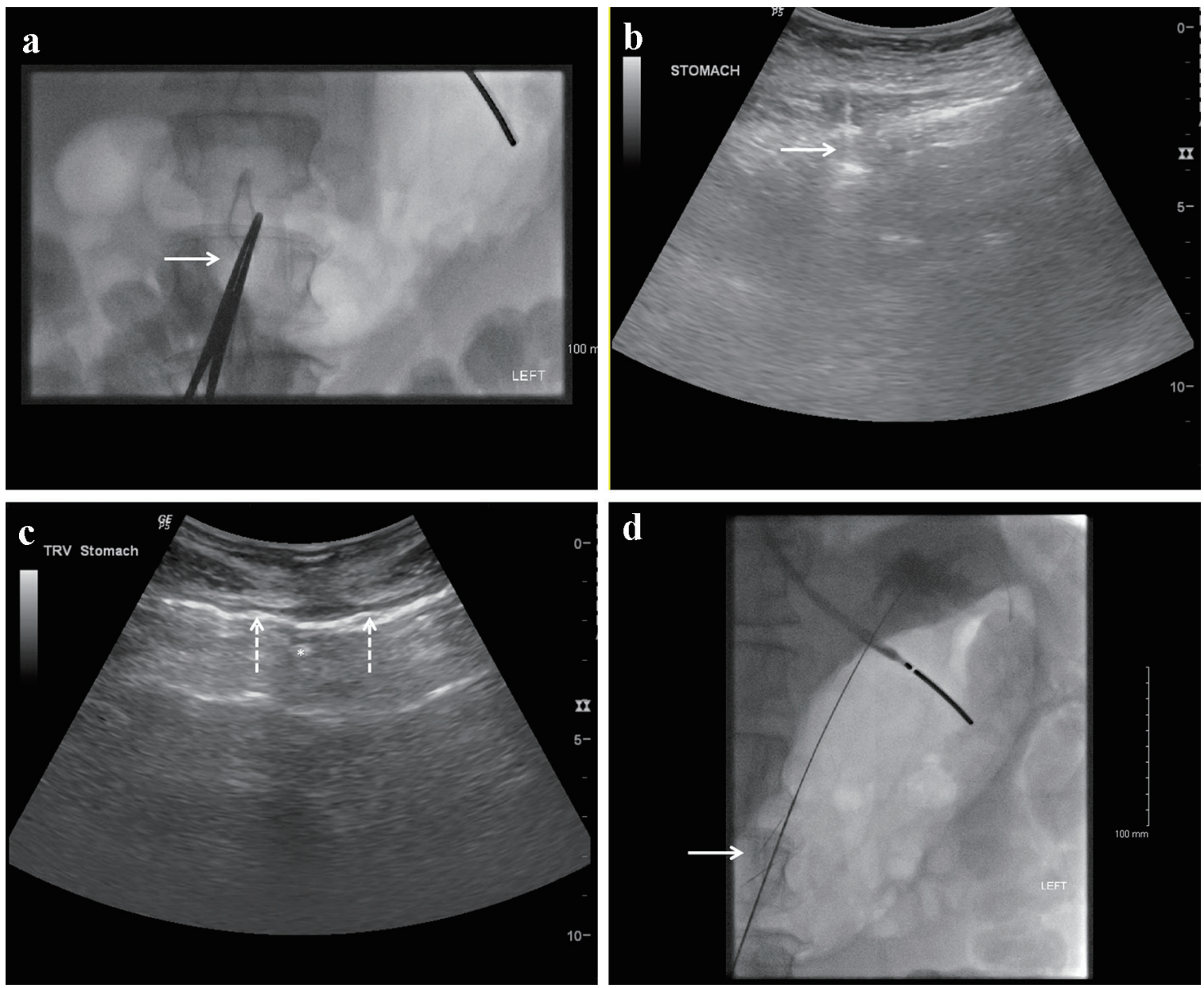

Figure 2. (a) Fluoroscopic image of the stomach insufflated with carbon dioxide with a superimposed clamp (arrow) on the patient's skin surface to mark the area for ultrasound guided antral access (note the distal end of the enteric tube seen in the fundus of the stomach). (b) Ultrasound guided placement of gastropexy through the rectus abdominal muscle lateral to the linea alba. (c) Ultrasound guided gastric access with an 18-gauge needle through the linea alba. *Gastric access needle tip. Gastropexy anchors anchoring the stomach to the anterior abdominal wall are denoted by arrows on both sides of the access needle. (d) Fluoroscopic image demonstrating a stiff guide wire through the access needle in between the gastropexy suture anchors (arrow) angled towards the fundus.

Fluoroscopy times and radiation doses were available for $30 / 31$ cases. A single case had concurrent placement of central venous access and only the cumulative fluoroscopy time and radiation dose were available, and was thus excluded from the following analyses. Average fluoroscopy time for all cases was $2.7 \pm 1.4 \mathrm{~min}$ and average radiation dose was $220 \pm 202 \mu \mathrm{Gm}^{2}$. Seven cases required nasogastric catheter placement for insufflation; the average fluoroscopy time and radiation dose for these cases was $4.1 \pm 1.4 \mathrm{~min}$ and $350 \pm 275 \mu \mathrm{Gm}^{2}$. When patients presented to the interventional radiology suite with a nasogastric or orogastric tube in place, the average fluoroscopy time and radiation dose were significantly less $(2.2 \pm 1.0 \mathrm{~min}$, $\left.\mathrm{P}<0.01 ; 180 \pm 163 \mu \mathrm{Gm}^{2}, \mathrm{P}<0.05\right)$.

\section{Discussion}

Percutaneous G-tube placement with endoscopic or radiologic guidance has largely replaced surgical placement of $G$ tubes due to the decreased incidence of post-procedure complications and need for general anesthesia. Although rare, procedural complications related to percutaneous placement of $\mathrm{G}$ tubes via fluoroscopic guidance have been documented. Inadvertent hepatic puncture may occur if the left hepatic lobe is near or crosses the midline [8]. At our institution, ultrasound of the abdomen is performed prior to insertion of a percutaneous $G$ tube by the operating interventional radiologist to identify and mark the liver margins. A slightly more frequent complication of percutaneous G-tube placement is colonic perforation due to proximity of the transverse colon to the gastric antrum [5]. When available, pre-procedure imaging (i.e. CT abdomen) is reviewed prior to each procedure for planning purposes. If not available, $\mathrm{CT}$ with oral contrast is only requested in patients in whom an altered anatomy is suspected (i.e. post-surgical abdomen).

Ultrasound assistance offers several benefits to fluorosco- 
py alone. The margins of the liver can be accurately identified and marked to prevent hepatic injury. In addition, real-time visualization of the needle entering the gastric antrum avoids inadvertent puncture of other organs. Ultrasound-guided access to the gastric antrum has been previously described. Lorentzen et al (2007) demonstrated safety and feasibility of ultrasound-guided access to the gastric antrum using water to distend the gastric lumen and create a fluid filled target for needle access in 154 patients [6]. The authors did not use ultrasound guidance for patients who could not drink water or have water infused using a nasogastric tube. However, 12 patients $(8 \%)$ in this study had post-procedure pneumonia, which may have been a result of aspiration especially in the setting of conscious sedation or general anesthesia. Bleck et al (1998) describe a technique in which water was infused into the stomach percutaneously with a small needle in patients that could not tolerate nasogastric tube placement due to severe stenosis [9].

We describe a technique achieving gastric antral access with ultrasound guidance and fluoroscopic confirmation following stomach insufflation with carbon dioxide [10]. Glucagon was administered to limit bowel motility and retain air within the stomach, permitting for ultrasound visualization of the gastric air bubble. Heberlein et al (2012) describe a technique of fluoroscopic puncture of the gastric bubble in patients in whom an enteric tube could not be placed; all patients were required to drink oral contrast prior to the procedure and effervescent granules were administered to a subset of those patients in attempts to distend the gastric air bubble as in an upper gastrointestinal series [11]. Attempts to access a nondistended stomach under ultrasound with subsequent insufflation air have also been described in patients in whom an enteric tube could be placed $[12,13]$.

A cross-table lateral fluoroscopy image may be obtained to identify interposition of colon between the stomach and abdominal wall. Pre-procedure oral contrast or barium enemas administered prior to G-tube insertion has been described for identification of the colon prior to puncture [8]. However, radiation exposure in fluoroscopic guided cases has become an area of interest in order to develop diagnostic reference levels to for quality assurance and improvement $[14,15]$. Percutaneous G-tube placement is considered a "low-dose" procedure with short fluoroscopy times and radiation doses [14]. However, it should be noted that standard deviation in fluoroscopy times and radiation doses can be high, which offers opportunity for improvements in technique. For example, in cases where barium is administered to patients for colonic identification, radiation dose is increased due to automatic exposure control [10]. In our study, mean fluoroscopy time was $2.7 \pm 1.4 \mathrm{~min}$. However, many of these patients required fluoroscopic cannulation of the gastroesophageal junction with a 4-F glide catheter because a nasogastric tube could not be passed through an alimentary tract stenosis. Subgroup analysis of patients that required fluoroscopic placement of a glide catheter shows a significant increase in fluoroscopy time and radiation dose.

CT fluoroscopy has been described for G-tube placement, which allows for real-time visualization of the stomach and surrounding organs (liver, colon, etc.) [16]. The authors emphasize the correlation with post-procedure complications to number of attempted accesses, which may be decreased with CT guidance. In our study, we demonstrate no procedure-related complications; however, this may be the result of a small sample size. Other limitations include those inherent to the retrospective nature of the study. Furthermore, all procedures were performed at a single institution by a single attending physician. No comparative data (i.e. fluoroscopic access) were included.

In conclusion, ultrasound guidance for gastric antral access is safe and effective for the placement of retrograde transabdominal gastrostomy tubes and may help reduce fluoroscopic time and radiation dose exposure.

\section{Acknowledgments}

None.

\section{Financial Disclosure}

None.

\section{Conflict of Interest}

On behalf of all authors, the corresponding author states that there is no conflict of interest.

\section{Informed Consent}

Informed consent was obtained from each patient.

\section{Author Contributions}

PS: data analysis, manuscript preparation. MK: data analysis, manuscript preparation. RT: data collection/analysis. AZ: data collection/analysis. AK: manuscript preparation. RP: principle investigator, project conception/design, and manuscript preparation.

\section{References}

1. Ho SG, Marchinkow LO, Legiehn GM, Munk PL, Lee MJ. Radiological percutaneous gastrostomy. Clin Radiol. 2001;56(11):902-910.

2. Wollman B, D'Agostino HB, Walus-Wigle JR, Easter DW, Beale A. Radiologic, endoscopic, and surgical gastrostomy: an institutional evaluation and meta-analysis of the literature. Radiology. 1995;197(3):699-704.

3. Haber ZM, Charles HW, Gross JS, Pflager D, Deipolyi AR. Percutaneous radiologically guided gastrostomy tube placement: comparison of antegrade transoral and retrograde transabdominal approaches. Diagn Interv Radiol. 2017;23(1):55-60. 
4. Cruz I, Mamel JJ, Brady PG, Cass-Garcia M. Incidence of abdominal wall metastasis complicating PEG tube placement in untreated head and neck cancer. Gastrointest Endosc. 2005;62(5):708-711; quiz 752, 753.

5. Covarrubias DA, O'Connor OJ, McDermott S, Arellano RS. Radiologic percutaneous gastrostomy: review of potential complications and approach to managing the unexpected outcome. AJR Am J Roentgenol. 2013;200(4):921-931.

6. Lorentzen T, Nolsoe CP, Adamsen S. Percutaneous radiologic gastrostomy with a simplified gastropexy technique under ultrasonographic and fluoroscopic guidance: experience in 154 patients. Acta Radiol. 2007;48(1):13-19.

7. Itkin M, DeLegge MH, Fang JC, McClave SA, Kundu S, Janne d'Othee B, Martinez-Salazar GM, et al. Multidisciplinary practical guidelines for gastrointestinal access for enteral nutrition and decompression from the Society of Interventional Radiology and American Gastroenterological Association (AGA) Institute, with endorsement by Canadian Interventional Radiological Association (CIRA) and Cardiovascular and Interventional Radiological Society of Europe (CIRSE). J Vasc Interv Radiol. 2011;22(8):1089-1106.

8. Ganeshan D. Re: Liver abscess after inadvertent transhepatic transgression during percutaneous fluoroscopyguided gastrostomy. Clin Radiol. 2009;64(1):105.

9. Bleck JS, Reiss B, Gebel M, Wagner S, Strassburg CP, Meier PN, Boozari B, et al. Percutaneous sonographic gastrostomy: method, indications, and problems.
Am J Gastroenterol. 1998;93(6):941-945.

10. Kelly B, Mullen E, Connolly B. Radiologic retrograde gastrostomy tube insertions without the use of barium: implications for radiation dose in children. AJR Am J Roentgenol. 2015;205(5):1135-1138.

11. Heberlein WE, Goodwin WJ, Wood CE, Yousaf M, Culp WC. Gastrostomy tube placement without nasogastric tube: a retrospective evaluation in 85 patients. Cardiovasc Intervent Radiol. 2012;35(6):1433-1438.

12. Inaba Y, Yamaura H, Sato Y, Kashima M, Kato M, Inoue D, Kurinobu T, et al. Percutaneous radiologic gastrostomy in patients with malignant pharyngoesophageal obstruction. Jpn J Clin Oncol. 2013;43(7):713-718.

13. Quadri A, Umapathy N, Orme R. Percutaneous gastrostomy in patients with complete obstruction of the upper digestive tract. Eur J Radiol. 2005;56(1):74-77.

14. Kloeckner R, Bersch A, dos Santos DP, Schneider J, Duber C, Pitton MB. Radiation exposure in nonvascular fluoroscopy-guided interventional procedures. Cardiovasc Intervent Radiol. 2012;35(3):613-620.

15. Pitton MB, Kloeckner R, Schneider J, Ruckes C, Bersch A, Duber C. Radiation exposure in vascular angiographic procedures. J Vasc Interv Radiol. 2012;23(11):14871495.

16. Tamura A, Kato K, Suzuki M, Sone M, Tanaka R, Nakasato T, Ehara S. CT-guided percutaneous radiologic gastrostomy for patients with head and neck cancer: a retrospective evaluation in 177 patients. Cardiovasc Intervent Radiol. 2016;39(2):271-278. 